\section{Cyclopentadienyl Titanium(IV) Complexes with Phenylisocyanate}

G. K. Parashar, P. C. Bharara, and R. C. Mehrotra

Department of Chemistry, University of Delhi, Delhi-110007

Z. Naturforsch. 34b, 109-110 (1979); received October 2,1978

Cyclopentadienyl Titanium Trialkoxides, Phenylisocyanate

Complexes of the type

$\mathrm{CpTi}(\mathrm{OR})_{3-n}[\mathrm{~N}(\mathrm{Ph}) \mathrm{COOR}]_{n}$ where $\mathrm{R}=\mathrm{Et}, \mathrm{Pr}^{i}$ and $\mathrm{Bu}^{t}, n=1,2$ or 3 , have been synthesized by the reactions of cyclopentadienyl titanium trialkoxides with phenylisocyanate. All these complexes have been characterized by the elemental analysis and infrared spectra.

Insertion across metal-oxygen bond is a topic of recent investigations and has been reported for a number of metal-alkoxides [1-3]. Meth-Cohn et al. [4] have studied the insertion into Ti-O bonds in $\mathrm{Ti}(\mathrm{OR})_{4}$. Very recently Choukroun et al. [5] have investigated the insertion reactions in $\left(\mathrm{Et}_{2} \mathrm{~N}\right) \mathrm{Ti}(\mathrm{OR})_{3}$. However, no reactions of this type have been reported for $\mathrm{CpTi}(\mathrm{OR})_{3}$. Since the introduction of a $\mathrm{Cp}$ group in $\mathrm{CpTi}(\mathrm{OR})_{3}$ should lead to greater $\mathrm{Ti}-\mathrm{O}$ bond covalent character, it was considered of

Requests for reprints should be sent to Prof. R. C. Mehrotra, Department of Chemistry, University of Delhi, Delhi-110007, India. interest to study the insertions in cyclopentadienyl titanium trialkoxides.

Synthesis of cyclopentadienyl titanium trialkoxides were carried out by reacting $\mathrm{CpTiCl}_{3}$ with corresponding alcohols in the presence of triethylamine [5]. For insertion reactions, weighed amounts of $\mathrm{CpTi}(\mathrm{OR})_{3}$ were placed in a flask to which benzene $(10 \mathrm{ml})$ was added. To this solution was mixed the phenylisocyanate in required molar ratios. Mixtures were set aside for about an hour and the completion of the reaction was checked by the disappearance of the infrared band at $\sim 2250 \mathrm{~cm}^{-1}$. The reaction products were finally dried by removing volatile fraction under reduced pressure.

The reactions were carried out under strictly anhydrous conditions and may be represented by the following general equation:

$$
\begin{aligned}
& \mathrm{CpTi}(\mathrm{OR})_{3}+n \mathrm{PhNCO} \rightarrow \\
& \mathrm{CpTi}(\mathrm{OR})_{3-n}[\mathrm{~N}(\mathrm{Ph}) \mathrm{COOR}]_{n} \\
& \text { where } \mathrm{R}=\mathrm{Et}, \mathrm{Pr}^{\mathrm{i}} \text { and } \mathrm{Bu}^{\mathrm{t}} ; n=1,2 \text { or } 3 .
\end{aligned}
$$

All the reactions were found exothermic in nature when PhNCO was added to a benzene solution of $\mathrm{CpTi}(\mathrm{OR})_{3}$. Reactions in $1: 1$ and $1: 2$ molar ratios were fast and facile as compared to that in the $1: 3$ molar ratio. Addition products

$\mathrm{CpTi}(\mathrm{OR})_{3-n}[\mathrm{~N}(\mathrm{Ph}) \mathrm{COOR}]_{n}$ thus isolated, were examined by their infrared spectral studies. In the infrared spectra of these complexes, no band was found in the region $\sim 2250 \mathrm{~cm}^{-1}$ which is a characteristic of $(-\mathrm{N}=\mathrm{C}=\mathrm{O})$ group in phenylisocyanate. Futhermore, the appearance of a new absorption band at $\sim 1710 \mathrm{~cm}^{-1}$ due to $v(\mathrm{C}=\mathrm{O})$ provides strong evidence that the insertion has taken place at the

\begin{tabular}{|c|c|c|c|c|}
\hline \multirow{2}{*}{$\begin{array}{l}\text { Reactants } \\
{[\mathrm{g}]}\end{array}$} & & \multirow[t]{2}{*}{ Products } & \multicolumn{2}{|c|}{ Found (Calcd) $[\%]$} \\
\hline & & & $\mathrm{Ti}$ & $\mathrm{N}$ \\
\hline $\begin{array}{l}\text { CpTi(OEt) } \\
\text { PhNCO }\end{array}$ & $\begin{array}{l}(0.437) \\
(0.209)\end{array}$ & $\mathrm{CpTi}(\mathrm{OEt})_{2}[\mathrm{~N}(\mathrm{Ph}) \mathrm{COOEt}]$ & $\begin{array}{c}13.1 \\
(13.0)\end{array}$ & $\begin{array}{c}3.7 \\
(3.8)\end{array}$ \\
\hline $\begin{array}{l}\text { CpTi(OEt) })_{3} \\
2 \text { PhNCO }\end{array}$ & $\begin{array}{l}(0.385) \\
(0.373)\end{array}$ & $\mathrm{CpTi}(\mathrm{OEt})[\mathrm{N}(\mathrm{Ph}) \mathrm{COOEt}]_{2}$ & $\begin{array}{c}9.65 \\
(9.85)\end{array}$ & $\begin{array}{c}5.6 \\
(5.7)\end{array}$ \\
\hline $\begin{array}{l}\text { CpTi(OEt) } \\
3 \text { PhNCO }\end{array}$ & $\begin{array}{l}(0.537) \\
(0.776)\end{array}$ & $\mathrm{CpTi}[\mathrm{N}(\mathrm{Ph}) \mathrm{COOEt}]_{3}$ & $\begin{array}{l}7.85 \\
(7.9)\end{array}$ & $\begin{array}{c}6.8 \\
(6.9)\end{array}$ \\
\hline $\begin{array}{l}\text { CpTi }\left(\mathrm{OPr}^{i}\right)_{3} \\
\text { PhNCO }\end{array}$ & $\begin{array}{l}(0.458) \\
(0.190)\end{array}$ & $\mathrm{Cp} T i\left(\mathrm{OPr}^{i}\right)_{2}\left[\mathrm{~N}(\mathrm{Ph}) \mathrm{COOPr}^{\imath}\right]$ & $\begin{array}{l}11.8 \\
(12.0)\end{array}$ & $\begin{array}{c}3.4 \\
(3.5)\end{array}$ \\
\hline $\begin{array}{l}\mathrm{CpTi}\left(\mathrm{OPr}^{i}\right)_{3} \\
2 \mathrm{PhNCO}\end{array}$ & $\begin{array}{l}(0.347) \\
(0.290)\end{array}$ & $\mathrm{Cp} \operatorname{Ti}\left(\mathrm{OPr}^{i}\right)\left[\mathrm{N}(\mathrm{Ph}) \mathrm{COOPr}^{i}\right]_{2}$ & $\begin{array}{l}9.15 \\
(9.2)\end{array}$ & $\begin{array}{c}5.3 \\
(5.4)\end{array}$ \\
\hline $\begin{array}{l}\mathrm{CpTi}\left(\mathrm{OPr}^{i}\right)_{3} \\
3 \mathrm{PhNCO}\end{array}$ & $\begin{array}{l}(0.504) \\
(0.623)\end{array}$ & $\mathrm{CpTi}\left[\mathrm{N}(\mathrm{Ph}) \mathrm{COOPr}^{i}\right]_{3}$ & $\begin{array}{l}7.45 \\
(7.5)\end{array}$ & $\begin{array}{c}6.5 \\
(6.5)\end{array}$ \\
\hline $\begin{array}{l}\mathrm{CpTi}\left(\mathrm{OBu}^{t}\right)_{3} \\
\text { PhNCO }\end{array}$ & $\begin{array}{l}(0.344) \\
(0.123)\end{array}$ & $\mathrm{CpTi}\left(\mathrm{OBu}^{t}\right)_{2}\left[\mathrm{~N}(\mathrm{Ph}) \mathrm{COOBu}^{t}\right]$ & $\begin{array}{c}10.4 \\
(10.6)\end{array}$ & $\begin{array}{l}2.95 \\
(3.1)\end{array}$ \\
\hline $\begin{array}{l}\mathrm{CpTi}\left(\mathrm{OBu}^{t}\right)_{3} \\
3 \mathrm{PhNCO}\end{array}$ & $\begin{array}{l}(0.402) \\
(0.432)\end{array}$ & $\mathrm{CpTi}\left[\mathrm{N}(\mathrm{Ph}) \mathrm{COOBu}^{t}\right]_{3}$ & $\begin{array}{c}6.8 \\
(6.9)\end{array}$ & $\begin{array}{c}6.0 \\
(6.1)\end{array}$ \\
\hline
\end{tabular}
$-\mathrm{N}=\mathrm{C}$ site.

Table I. Reactions of CpTi(OR $)_{3}$ with PhNCO. 
[1] R. C. Mehrotra, A. K. Rai, and R. Bohra, J. Inorg. Nucl. Chem. 36, 1887 (1974).

[2] R. C. Mehrotra, V. D. Gupta, and P. C. Bharara, Indian J. Chem. 13, 156 (1975).

[3] R. C. Mehrotra, A. K. Rai, and R. Bohra, Syn. React. Inorg. Metal-Org. Chem. 5, 289 (1975).
[4] O. Meth-Cohn, D. Thorpe, and H. J. Twitchett, J. Chem. Soc. C. 1970, 132 .

[5] R. Choukroun and D. Gervais, Syn. React. Inorg. Metal-Org. Chem. 8, 137 (1978).

[6] A. C. Skapski, P. G. H. Throughton, and H. H. Sutherland, Chem. Commun. 1968, 1418. 